\title{
SOME MARKOVIAN PROPERTIES OF LINEAR DIFFERENTIAL SYSTEMS*
}

\author{
JIANGFENG ZHANG ${ }^{\dagger}$ AND C.P. KWONG KH $^{\prime}$
}

\begin{abstract}
In this paper we study the Markovian properties of a system of linear partial differential equations with constant coefficients as initiated by J.C. Willems. In particular, we prove that his conjecture on Markovianity is true if the characteristic variety of the system has dimension zero. For the case when the system is defined by a differential operator, we give conditions under which the conjecture is also valid.
\end{abstract}

Key word: Linear differential systems, Markovianity, characteristic variety.

1. Introduction. The behavioral approach developed by J.C. Willems ([11]) for systems determined by linear constant coefficient partial differential equations (PDEs) has attracted research interest recently ([8], [12]). In [12] a general definition of Markovianity is given in order to study the concept of states and their construction for $n$-dimensional systems. A conjecture has also been posed, namely that the behavior of a system is Markovian if and only if it has a kernel representation that is first order.

In this paper we will use the Ehrenpreis-Palamodov Fundamental Principle in the study of PDEs (see [3], [6], [7], or Chapter 8 of [1] for an introduction) to show that the above conjecture is true if the characteristic variety (to be defined in the next section) of the system is of dimension zero. A system with zero-dimensional characteristic variety has finite-dimensional behavior. We will show that the conjecture is also valid for some special infinite-dimensional behaviors.

Whereas the formulation of the Willems' conjecture aims to understand the properties of the behavior of a PDE system, our result indicates that the behavior is defined by a linear space with a finite basis when the characteristic variety of the system is of dimension zero. It follows that the behavior will become transparent if each component of this finite basis can be obtained explicitly. With the introduction of the notion of characteristic variety this computational problem becomes feasible. We will show by an example how to apply the theory of Gröbner bases in commutative algebra (see [2] for an introduction) to determine whether the characteristic variety of a system has dimension zero, and if it is the case, to compute the relevant basis.

The paper is organized as follows. In Section 2 we review the behavioral approach

\footnotetext{
*This work was supported by a grant from the Research Grants Council of Hong Kong (Project CUHK4185/01E).

$\dagger$ Department of Automation and Computer-Aided Engineering, The Chinese University of Hong Kong, Shatin, N.T., Hong Kong., E-mail: jfzhangjf@yahoo.com.cn

${ }^{\ddagger}$ Corresponding author. Department of Automation and Computer-Aided Engineering, The Chinese University of Hong Kong, Shatin, N.T., Hong Kong., E-mail: cpkwong@acae.cuhk.edu.hk
} 
to the study of differential systems. The definition of a characteristic variety and relevant results of PDEs are also given in this section for preparing the derivation of our main results in the next section, and the presentation of a computational example in Section 4.

2. Preliminaries. We first give a brief review of the behavioral theory of Willems and his conjecture ([12]). Let $x=\left(x_{1}, x_{2}, \ldots, x_{n}\right)$ be $n$-tuple of real independent variables and $\mathcal{D}^{\prime}(X)$ the set of real distributions on $X \subset \mathbb{R}^{n}$. We also write $\mathcal{D}^{\prime}$ for $\mathcal{D}^{\prime}\left(\mathbb{R}^{n}\right)$. Suppose $\mathcal{L}_{n}^{w}$ is the set of linear subspaces of $\left(\mathcal{D}^{\prime}\right)^{w}$ consisting of the solutions of a system of linear constant coefficient PDEs, i.e., each element $\mathcal{B} \in \mathcal{L}_{n}^{w}$ is defined by a polynomial matrix $R \in \mathbb{R}^{\bullet \times w}\left[\xi_{1}, \xi_{2}, \ldots, \xi_{n}\right]$ with $w$ columns but any number of rows, such that

$$
\mathcal{B}=\left\{u \in\left(\mathcal{D}^{\prime}\right)^{w} \mid R\left(\frac{\partial}{\partial x_{1}}, \frac{\partial}{\partial x_{2}}, \ldots, \frac{\partial}{\partial x_{n}}\right) u=0\right\} .
$$

A member of $\mathcal{L}_{n}^{w}$ is called a linear differential $n$-D system. The above PDE is called a kernel representation of the behavior $\mathcal{B} \in \mathcal{L}_{n}^{w}$.

Denote by $\Delta$ the following set of partitions of $\mathbb{R}^{n}$ :

$$
\begin{aligned}
{\left[\left(S_{-}, S_{0}, S_{+}\right) \in \Delta\right] \Leftrightarrow \quad } & {\left[( S _ { - } , S _ { 0 } , S _ { + } \text { are disjoint subsets of } \mathbb { R } ^ { n } ) \wedge \left(S_{-} \cup S_{0} \cup\right.\right.} \\
& \left.\left.S_{+}=\mathbb{R}^{n}\right) \wedge\left(S_{-} \text {and } S_{+} \text {are open, and } S_{0} \text { is closed }\right)\right] .
\end{aligned}
$$

For any subset $\mathcal{F}$ of $\mathbb{R}^{w}$ or $\mathbb{C}^{w}$, and any $f_{-}, f_{+}: \mathbb{R}^{n} \rightarrow \mathcal{F}$, let $\pi=\left(S_{-}, S_{0}, S_{+}\right) \in \Delta$. The concatenation of $\left(f_{-}, f_{+}\right)$along $\pi$ is the map $f_{-} \wedge_{\pi} f_{+}: \mathbb{R}^{n} \longrightarrow \mathcal{F}$ defined by

$$
f_{-} \wedge_{\pi} f_{+}(x)=\left\{\begin{array}{l}
f_{-}(x) \text { for } x \in S_{-} \\
f_{+}(x) \text { for } x \in S_{0} \cup S_{+} .
\end{array}\right.
$$

A behavior $\mathcal{B} \in \mathcal{L}_{n}^{w}$ is called Markovian if

$$
\begin{aligned}
& {\left[\left(u_{-}, u_{+} \in \mathcal{B} \cap C^{\infty}\left(\mathbb{R}^{n}, \mathbb{R}^{w}\right)\right) \wedge\left(\pi=\left(S_{-}, S_{0}, S_{+}\right) \in \Delta\right) \wedge\left(\left.u_{-}\right|_{S_{0}}=\left.u_{+}\right|_{S_{0}}\right)\right] } \\
\Rightarrow \quad & {\left[u_{-} \wedge_{\pi} u_{+} \in \mathcal{B}\right] . }
\end{aligned}
$$

A Markovian behavior $\mathcal{B}$ is nontrivial if there exist two distinct elements $u_{-}, u_{+} \in$ $\mathcal{B} \cap C^{\infty}\left(\mathbb{R}^{n}, \mathbb{R}^{w}\right)$ such that $\left.u_{-}\right|_{S_{0}}=\left.u_{+}\right|_{S_{0}}$ for some partition $\pi=\left(S_{-}, S_{0}, S_{+}\right)$. Note that we only consider nontrivial Markovian behaviors in this paper.

By the linearity of kernel representation it is easy to prove the following result.

Proposition 1. A behavior $\mathcal{B} \in \mathcal{L}_{n}^{w}$ is Markovian if and only if for any $u \in$ $\mathcal{B} \cap C^{\infty}\left(\mathbb{R}^{n}, \mathbb{R}^{w}\right)$ such that $\left.u\right|_{S_{0}}=0$ for some partition $\pi=\left(S_{-}, S_{0}, S_{+}\right)$of $\mathbb{R}^{n}$, we have $u \wedge_{\pi} 0 \in \mathcal{B}$.

Markovianity has an obvious symmetry as shown in the following proposition.

Proposition 2. Suppose a behavior $\mathcal{B}$ is Markovian and nontrivial, then both $u_{-} \wedge_{\pi} u_{+}$and $u_{+} \wedge_{\pi} u_{-}$belong to $\mathcal{B}$. 
Willems proposes the following conjecture in [12].

Conjecture. The behavior $\mathcal{B} \in \mathcal{L}_{n}^{w}$ is Markovian if and only if it has a kernel representation that is first order, i.e., it has the following kernel representation

$$
R_{0} u+R_{1} \frac{\partial}{\partial x_{1}} u+R_{2} \frac{\partial}{\partial x_{2}} u+\cdots+R_{n} \frac{\partial}{\partial x_{n}} u,
$$

where $R_{i}, i=0,1, \ldots, n$, are constant matrices.

The validity of the conjecture for $n=1$ has been proved in [9]. The sufficient part for general $n$ is straightforward to prove ([12]) while the necessary part remains open.

For a behavior defined by an $s \times w$ polynomial matrix $R=\left(R_{j k}(x)\right)$ where $R_{j k}(x)=R_{j k}\left(x_{1}, x_{2}, \ldots, x_{n}\right)$ are polynomials in $n$ variables, we denote the PDE system corresponding to $R$ by $R(D)=0$ or $R(D) u=0$. The characteristic variety of this system (or behavior) is defined by the following set

$$
V_{R}=\left\{\tau \in \mathbb{C}^{n}: \text { the matrix }\left(R_{j k}(\tau)\right) \text { has rank }<w\right\} .
$$

It is clear that if $s<w$ then $V_{R}=\mathbb{C}^{n}$ - the under-determined case in which there are more unknowns than equations. In this paper we consider only the more interesting over-determined case, i.e., $s \geq w$.

By definition the characteristic variety of $R$ characterizes the common solution of a system of polynomials where each polynomial is a $w \times w$ sub-determinant of $R$. Much useful information about the behavior of a system can be obtained from its characteristic variety. An example is the following proposition, which is an easy variation of Proposition 8.1.8 in [1].

Proposition 3. With notations given above, let $\tau=\left(\tau_{1}, \tau_{2}, \ldots, \tau_{n}\right) \in V_{R}$, then there exist complex scalars $c_{1}, c_{2}, \ldots, c_{w}$ of which at least one is nonzero, such that $\left(c_{1} e^{(x, \tau)}, c_{2} e^{(x, \tau)}, \ldots, c_{w} e^{(x, \tau)}\right)$ is an exponential solution of $R(D) u=0$, where $(x, \tau)$ denotes the usual Euclidean inner product.

Next we introduce the Ehrenpreis-Palamodov Fundamental Principle for the case $\operatorname{dim}\left(V_{R}\right)=0$, i.e., $V_{R}$ has only a finite number of elements in $\mathbb{C}^{n}$. The Principle is an important result for the integral representation of solutions of linear PDEs with constant coefficients.

Theorem 1. ([1], Theorem 8.7.1) Suppose $\operatorname{dim}\left(V_{R}\right)=0$, then there exists a finite set of exponential solutions $\chi_{j}=\left(q_{j 1}(x) e^{\left(x, \tau_{j}\right)}, q_{j 2}(x) e^{\left(x, \tau_{j}\right)}, \ldots, q_{j w}(x) e^{\left(x, \tau_{j}\right)}\right)$, where $\left\{q_{j k}(x)\right\}$ are some polynomials in $x_{1}, x_{2}, \ldots, x_{n}$ and $\tau_{j}$ 's belong to $V_{R}$ such that the following is true: If $\Omega$ is some open subset of $\mathbb{R}^{n}$ and if $\mu=\left(\mu_{1}, \mu_{2}, \ldots, \mu_{w}\right)$ is some wtuple of distributions in $\Omega$ satisfying $R(D) \mu=0$ in $\Omega$, then there exist complex scalars $\left\{c_{j}\right\}$ such that $\mu=\sum c_{j} \chi_{j}$, i.e., each distribution $\mu_{k}$ is of the form $\sum c_{j} q_{j k}(x) e^{\left(x, \tau_{j}\right)}$ and thus the locally defined solution $\mu$ extends to an exponential solution in the whole space $\mathbb{R}^{n}$. 
The above results describe the solutions for a finite-dimensional behavior. As for infinite-dimensional behaviors, a simple case is that the matrix $R$ has only one element, that is, the behavior is modeled by a single differential operator $P(D)$. In this case the following results [5] are useful.

Suppose a differential operator $P(D)$ is defined by a polynomial

$$
P(x)=\sum_{|\alpha| \leq m} c_{\alpha} x^{\alpha}
$$

with degree $m$ where $\alpha=\left(\alpha_{1}, \alpha_{2}, \ldots, \alpha_{n}\right)$ is a multi-index. Define the principal part of $P$ by $P_{m}=\sum_{|\alpha|=m} c_{\alpha} x^{\alpha}$. If $\xi \in \mathbb{R}^{n}$ is a nonzero solution of $P_{m}(x)=0$, then $\xi$, or the hyperplane which has $\xi$ as its normal, is called a characteristic of the differential operator $P(D)$.

Theorem 2. ([5], Theorem 8.6.8) Let $X_{1}$ and $X_{2}$ be open convex sets in $\mathbb{R}^{n}$ such that $X_{1} \subset X_{2}$, and $P(D)$ be defined as above, then the following statements are equivalent.

(i) Every $u \in \mathcal{D}^{\prime}\left(X_{2}\right)$ satisfying the equation $P(D) u=0$ in $X_{2}$ and vanishing in $X_{1}$ must also vanish in $X_{2}$.

(ii) Every hyperplane which is a characteristic with respect to $P$ and intersects $X_{2}$ also intersects $X_{1}$.

The differential operator $P(D)$ (and the polynomial $P(x)$ ) is called hypoelliptic if $P^{(\alpha)}(x) / P(x) \rightarrow 0$ as $x \rightarrow \infty$ in $\mathbb{R}^{n}$ for any nonzero multi-index $\alpha$, where $P^{(\alpha)}(x)$ denotes the partial derivative $D^{\alpha} P(x)$. Theorem 11.1.3 in [5] gives several equivalent algebraic conditions to check if an operator is hypoelliptic. The following proposition gives an important analytic condition. Note that elliptic operators and semi-elliptic operators are special cases of hypoelliptic operator.

Proposition 4. ([5], Theorem 11.1.1) The differential operator $P(D)$ is hypoelliptic if and only if, for any open set $X \subset \mathbb{R}^{n}, u \in \mathcal{D}^{\prime}(X)$, and $P(D) u=0$, we have $u \in C^{\infty}(X)$.

For the polynomial $P(x)$ and any linear subspace $V$ of $\mathbb{R}^{n}$, we define the following functions

$$
\begin{array}{ll}
\tilde{P}_{V}(\xi, t) & :=\sup \{|P(\xi+\theta)|: \theta \in V,|\theta| \leq t\} \\
\tilde{P}(\xi, t) & :=\tilde{P}_{\mathbb{R}^{n}}(\xi, t) \\
\sigma_{P}(V) & :=\inf _{t>1} \frac{\lim }{\xi \rightarrow \infty} \tilde{P}_{V}(\xi, t) / \tilde{P}(\xi, t) .
\end{array}
$$

Proposition 5. ([5], Theorem 11.3.6) Let $X$ be an open set in $\mathbb{R}^{n}, x_{0}$ a point in $X$ and $\phi_{1}, \phi_{2}, \ldots, \phi_{k} \in C^{1}(X)$ real valued functions such that $d \phi_{1}\left(x_{0}\right), d \phi_{2}\left(x_{0}\right), \ldots$, $d \phi_{k}\left(x_{0}\right)$ are linearly independent. Assume that $\sigma_{P}(W) \neq 0$ for the space $W$ spanned by $d \phi_{1}\left(x_{0}\right), d \phi_{2}\left(x_{0}\right), \ldots, d \phi_{k}\left(x_{0}\right)$ and let

$$
X_{-}=\left\{x \in X: \phi_{j}(x) \leq \phi_{j}\left(x_{0}\right) \text { for some } j=1,2, \ldots, k\right\} .
$$


If $u \in \mathcal{D}^{\prime}(X), P(D) u \in C^{\infty}(X)$, and $u \in C^{\infty}\left(X_{-}\right)$, then $u \in C^{\infty}$ in a neighborhood of $x_{0}$ which is independent of $u$.

Proposition 6. ([5], Theorem 11.3.13) Let $\Gamma$ be a closed convex set in $\mathbb{R}^{n}$ and $V$ the largest vector space with $\Gamma+V=\Gamma$. Then $u \in \mathcal{D}^{\prime}\left(\mathbb{R}^{n}\right), P(D) u=0$, and sing supp $u \subset \Gamma$ implies $u \in C^{\infty}\left(\mathbb{R}^{n}\right)$ if and only if $\sigma_{P}\left(V^{\prime}\right) \neq 0$, where sing supp $u$ is defined as the set of points in $\mathbb{R}^{n}$ having no open neighborhood to which the restriction of $u$ is a $C^{\infty}$ function, and $V^{\prime}$ is the subspace orthogonal to $V$.

3. The Willems' Conjecture. In this section we give our main results on the Willems' conjecture.

TheOREm 3. Let the matrix $R$ be defined as before and assume that the characteristic variety $V_{R}$ has dimension zero. If the behavior $\mathcal{B}$ defined by $R(D)$ is Markovian and nontrivial, then it has a kernel representation which is first order.

Proof. It follows from Theorem 1 that the behavior $\mathcal{B}$ has a finite basis $\chi_{j}=$ $\left(q_{j 1}(x) e^{\left(x, \tau_{j}\right)}, q_{j 2}(x) e^{\left(x, \tau_{j}\right)}, \ldots, q_{j w}(x) e^{\left(x, \tau_{j}\right)}\right)$ for $j=1,2, \ldots, t$ and each solution of the system is a linear combination of these $\chi_{j}$ 's. Suppose the kernel representation of the Markovian behavior is not first order, then there exists a nonzero solution $u$ which vanishes on $S_{0}$ for some partition $\pi=\left(S_{-}, S_{0}, S_{+}\right)$. By Proposition 1 the concatenation of $u$ and 0 belongs to $\mathcal{B}$. Consequently, the concatenation can be expressed as a linear combination of $\chi_{j}$ 's. Therefore we can write $u=\sum_{j=1}^{t} c_{j} \chi_{j}, u \wedge_{\pi}$ $0=\sum_{j=1}^{t} d_{j} \chi_{j}$, and

$$
\sum_{j=1}^{t} d_{j} \chi_{j}(x)= \begin{cases}\sum_{j=1}^{t} c_{j} \chi_{j}(x), & \text { for } x \in S_{-} \\ 0, & \text { for } x \in S_{+} .\end{cases}
$$

Since exponential functions are analytic at every point of $\mathbb{C}^{n}$, the function $\sum_{j=1}^{t} d_{j} \chi_{j}$ is analytic in $\mathbb{C}^{n}$ when $x$ is viewed as an $n$-tuple of complex variables. Notice that $\sum_{j=1}^{t} d_{j} \chi_{j}$ is identical to the analytic function 0 on the open set $S_{+}$. It follows that the identity holds for the entire $\mathbb{C}^{n}$ by the identity theorem of holomorphic functions (see, for example, Theorem 1.4.1 of [4]). Since we have assumed that $\left\{\chi_{j}\right\}$ is a basis, which means that the $\chi_{j}$ 's are linearly independent, then $d_{j}=0$ for $j=1,2, \ldots, t$. Hence $u=0$ for $x \in S_{-}$by virtue of the identity theorem. This clearly contradicts Markovianity, and the result follows.

Thus we have shown that the Willems' conjecture holds for finite-dimensional behaviors. In the following we consider a special kind of infinite-dimensional behaviors defined by the kernel of a linear differential operator with constant coefficients, that is, we consider the case when $R$ is a $1 \times 1$ matrix.

Let $P(D)$ be a differential operator and its corresponding polynomial $P(x)$ has degree $m \geq 2$. Suppose $P(x)$ has at least two distinct real zeros $\tau=\left(\tau_{1}, \tau_{2}, \ldots, \tau_{n}\right)^{T}$ and $\delta=\left(\delta_{1}, \delta_{2}, \ldots, \delta_{n}\right)^{T}$. It is clear that the two zeros define the following partition 
$\pi=\left(S_{-}, S_{0}, S_{+}\right)$of $\mathbb{R}^{n}:$

$$
\begin{aligned}
& S_{-}:=\left\{x \in \mathbb{C}^{n}:(\tau-\delta)^{T} x<0\right\}, \\
& S_{0}:=\left\{x \in \mathbb{C}^{n}:(\tau-\delta)^{T} x=0\right\}, \\
& S_{+}:=\left\{x \in \mathbb{C}^{n}:(\tau-\delta)^{T} x>0\right\} .
\end{aligned}
$$

Lemma 1. If the behavior $\mathcal{B}$ corresponds to the above differential operator $P(D)$ is Markovian, then the function

$$
u(x)= \begin{cases}0, & \text { if } x \in S_{-} ; \\ e^{(x, \tau)}-e^{(x, \delta)}, & \text { if } x \in S_{+} \cup S_{0}\end{cases}
$$

belongs to $\mathcal{B}$ and is not $C^{1}$-continuous, i.e., not all the first partial derivatives of $u(x)$ are continuous.

Proof. It is obvious that the solution set of $P(x)=0$ is just the characteristic variety of $P(D)$. Then Proposition 3 tells us that both $e^{(x, \tau)}$ and $e^{(x, \delta)}$ are solutions of $P(D) u=0$, and so is $e^{(x, \tau)}-e^{(x, \delta)}$. If the system is Markovian, then by Proposition $1, u(x) \in \mathcal{B}$ since $u(x)$ is just the concatenation of 0 and $\left(e^{(x, \tau)}-e^{(x, \delta)}\right)$ with respect to $\pi$.

Since $\tau$ and $\delta$ are distinct, we may suppose that $\tau_{k} \neq \delta_{k}$ for some $k$. Then the partial derivative $\frac{\partial u(x)}{\partial x_{k}}$ does not exist at 0 because the limits in $S_{-}$and $S_{+}$do not coincide. This completes the proof.

Theorem 4. Let $P(D)$ be a differential operator with degree $m \geq 2$. If the corresponding characteristic variety $V_{P}$ has at least two distinct real elements, then the behavior is not Markovian under either one of the following conditions.

(i) There exist two distinct real elements $\tau$ and $\delta$ of $V_{P}$ such that $\tau-\delta$ is not a characteristic of $P(D)$, i.e., $P_{m}(\tau-\delta) \neq 0$, where $P_{m}$ is the principal part of $P(D)$.

(ii) The operator $P(D)$ is hypoelliptic.

(iii) There exists two distinct real elements $\tau$ and $\delta$ of $V_{P}$ such that $\sigma_{P}(W) \neq 0$ or $\sigma_{P}\left(W^{\prime}\right) \neq 0$, where $W$ is the line spanned by $(\tau-\delta)$ in $\mathbb{R}^{n}$ and $W^{\prime}$ is the orthogonal subspace of $W$.

Proof. (i) In Theorem 2, let $X_{2}=\mathbb{R}^{n}, X_{1}=S_{-}$, where $S_{-}$is defined as in Lemma 1. It is obvious that every hyperplane in $\mathbb{R}^{n}$ except $S_{0}$ must pass through $S_{-}$. Since $S_{0}$ is not a characteristic of $P(D)$, the second statement of Theorem 2 holds and hence each $u \in \mathcal{B}$ that vanishes in $S_{-}$must vanish in $\mathbb{R}^{n}$. If $\mathcal{B}$ is Markovian, then by Lemma 1 , there exists a $u \in \mathcal{B}$ that is nonzero in $S_{+}$but vanishes in $S_{-}$, which is a contradiction. Thus the system is not Markovian.

(ii) Let $X=\mathbb{R}^{n}$ in Proposition 4 , then every $u \in \mathcal{B}$ must be $C^{\infty}$. However, Lemma 1 gives an explicit $u$ which does not belong to $C^{1}\left(\mathbb{R}^{n}\right)$ and hence the behavior associated with a hypoelliptic operator can not be Markovian.

(iii) Suppose the system is Markovian. For the case $\sigma_{P}(W) \neq 0$, we refer to Proposition 5 and let $k=1, \phi_{1}=\sum_{j=1}^{n} x_{j}\left(\tau_{j}-\delta_{j}\right), X=\mathbb{R}^{n}$, and $x_{0}=0$. Hence 
$W$ is the line spanned by $(\tau-\delta)$, and $X_{-}=S_{-} \cup S_{0}$ where $S_{-}$and $S_{0}$ are defined as in Lemma 1. The distribution $u$ defined in Lemma 1 satisfies all the conditions of Proposition 5 since $P(D) u=0 \in C^{\infty}\left(\mathbb{R}^{n}\right)$. Therefore $u \in C^{\infty}$ is in a neighborhood of 0 , which clearly contradicts the result of Lemma 1 . For the case $\sigma_{P}\left(W^{\prime}\right) \neq 0$, we refer to Proposition 6 and let $\Gamma=S_{0}$, where $S_{0}$ is the same as in Lemma 1. It is obvious that $V=S_{0}$ and the distribution $u$ defined in Lemma 1 has its singular support sing supp $u$ contained in $\Gamma$. Then $u \in C^{\infty}\left(\mathbb{R}^{n}\right)$, which contradicts the result of Lemma 1. This completes the proof.

Remark. Note that Condition (i) includes those differential operators which are hyperbolic with respect to $\tau-\delta$. All these three conditions cover a large class of differential operators. For instance, the behaviors of the following equations are not Markovian:

$$
\begin{array}{ll}
\frac{\partial^{2}}{\partial x_{1}^{2}} u+\frac{\partial^{2}}{\partial x_{2}^{2}} u+\cdots+\frac{\partial^{2}}{\partial x_{n}^{2}} u-a \frac{\partial}{\partial t} u & =0, \\
\frac{\partial^{2}}{\partial x_{1}^{2}} u+\frac{\partial^{2}}{\partial x_{2}^{2}} u+\cdots+\frac{\partial^{2}}{\partial x_{n}^{2}} u-\frac{1}{c^{2}} \frac{\partial^{2}}{\partial t^{2}} u & =0, \\
\frac{\partial^{2}}{\partial x_{1}^{2}} u+\frac{\partial^{2}}{\partial x_{2}^{2}} u+\cdots+\frac{\partial^{2}}{\partial x_{n}^{2}} u & =0 .
\end{array}
$$

4. Computation of Exponential Solutions. We have shown that if the dimension $\operatorname{dim} V_{R}$ of the characteristic variety of a system of PDEs is zero, then the behavior of the system, according to the Ehrenpreis-Palamodov Fundamental Principle, can be represented by a linear combination of exponentials. We will give in the following an example to show how we can determine algorithmically whether a characteristic variety is of dimension zero, and if it is the case, compute the exponential basis. The principle behind these computations is the theory of Gröbner bases in computational commutative algebra ([2]) and the software we use is Maple. Note that when $\operatorname{dim} V_{R}>0$, the characteristic variety contains infinite elements in $\mathbb{C}^{n}$ but possibly finite elements in $\mathbb{R}^{n}$. For this latter finite case all the real exponential solutions can be found.

EXAMPle. Consider the following system of PDEs:

$$
\begin{cases}\left(\frac{\partial^{2}}{\partial z^{2}}+\frac{\partial^{2}}{\partial y^{2}}+\frac{\partial}{\partial x}+1\right) u & =0 \\ \left(\frac{\partial^{2}}{\partial y^{2}}+\frac{\partial}{\partial z}-1\right) u & =0 \\ \left(\frac{\partial}{\partial x}+2 \frac{\partial}{\partial z}+\frac{\partial}{\partial y}\right) u & =0\end{cases}
$$

In this example the matrix $R$ is a $3 \times 1$ matrix and the characteristic variety is the solution set of the three polynomials in $R$ :

$$
V_{R}=\left\{(x, y, z) \in \mathbb{C}^{3} \mid z^{2}+y^{2}+x+1=0, y^{2}+z-1=0, x+2 z+y=0\right\} .
$$

We first use the Maple command 'hilbertdim' to find the dimension of this variety. The returned result is zero. Next, we use the command 'solve' to obtain the following 
solution:

$$
(x=-2, y=0, z=1),\left(x=2 a_{j}^{2}-2-a_{j}, y=a_{j}, z=-a_{j}^{2}+1\right), j=1,2,3,
$$

where $a_{j}$ are roots of $a^{3}+a-1=0, j=1,2,3$. The approximate values of these $a_{j}$ are

$$
\begin{aligned}
& -0.3411639019-1.161541400 \sqrt{-1},-0.3411639019+1.161541400 \sqrt{-1}, \\
& 0.6823278038
\end{aligned}
$$

Thus each solution is a simple zero and the following exponential solutions form a basis of the behavior of Equations (4.1).

$$
\begin{aligned}
& u_{0}=e^{-2 x+z} \\
& u_{j}=e^{\left(2 a_{j}^{2}-2-a_{j}\right) x+a_{j} y+\left(-a_{j}^{2}+1\right) z}, j=1,2,3 .
\end{aligned}
$$

It is also easy to check by definition that the above system is not Markovian. Note that when the characteristic variety has multiple points, the behavior is the product of an exponential function with a polynomial. A detailed discussion on the computation of these polynomials for $R$ with a single column can be found in [10]. Disregarding whether the characteristic variety $V_{R}$ has multiple points, as a finitedimensional complex vector space its dimension can be computed once we know the Gröbner basis of the polynomials. For example, in the above Equations (4.1), the Gröbner basis for $V_{R}$ with respect to the total degree order $z>y>x$ is

$\left\{x+2 z+y, 13 x+18+4 y x+2 x^{2}+5 y, 2 y^{2}-y-x-2,8 x^{3}+86 x^{2}-39 y+345 x+410\right\}$.

The command 'SetBasis' of Maple gives further the basis of the residue ring

$$
\mathbb{C}[x, y, z] / I:\left\{1, x, y, x^{2}\right\},
$$

where $I$ is the ideal generated by the three polynomials in $R$. Therefore $V_{R}$ is a fourdimensional complex vector space since the above basis has only four elements ([2], Theorem 5.3.6), and so is the behavior $\mathcal{B}$. Knowing the number of basis components of $\mathcal{B}$ will help us to find the missing polynomial-exponential solutions when $V_{R}$ has multiple points (see [10]).

5. Conclusions. We have shown in this paper that if the characteristic variety of a system of linear PDEs with constant coefficients has dimension zero, then the Willems' conjecture on the Markovianity of behavior is true. For a behavior defined by a differential operator, we have given several conditions under which the conjecture is valid. An example has been given to show how to determine, by using computer algebra software, whether the characteristic variety of a system is of dimension zero. In case the answer is affirmative, a finite basis of the behavior can be computed by the same software. 


\section{REFERENCES}

[1] J.-E. BJöRK, Rings of Differential Operators, North-Holland Publishing Company, Amsterdam, 1979.

[2] D. Cox, J. Little, And D. O’Shea, Ideals, Varieties and Algorithms, Second edition, Springer, New York, 1997.

[3] L. Ehrenpreis, Fourier Analysis in Several Complex Variables, Wiley-Interscience, New York, 1970.

[4] H. Grauert and K. Fritzsche, Several Complex Variables, Graduate Texts in Mathematics, Volume 38, Springer, New York, 1976.

[5] L. Hörmander, The Analysis of Linear Partial Differential Operators (I, II), Springer, New York, 1983.

[6] U. OBerst, Multidimensional constant linear systems, Acta Applicandae Mathematicae, 20(1990), pp. 1-175.

[7] V.P. Palamodov, Linear Differential Operators with Constant Coefficients, Springer, New York, 1970.

[8] H.K. Pillai and S. Shankar, A behavioral approach to control of distributed systems, SIAM Journal on Control and Optimization, 37(1999), pp. 388-408.

[9] P. Rapisarda and J.C. Willems, State maps for linear systems, SIAM Journal on Control and Optimization, 35(1997), pp. 1053-1091.

[10] B. Sturmfels, Solving Systems of Polynomial Equations, CBMS Regional Conference Series in Mathematics, American Mathematical Society, Providence, R.I., 2002.

[11] J.C. Willems, On interconnections, control, and feedback, IEEE Transactions on Automatic Control, 42(1997), pp. 326-339.

[12] J.C. Willems, State and first order representations, in: V.D. Blondel and A. Megretski (eds.), Open Problems on the Mathematical Theory of Systems, 2002, pp. 1-3. 
